\title{
Health-related quality of life in children and adolescents undergoing surgery for Hirschsprung's disease and anorectal malformations
}

\author{
Mariana Bazo ${ }^{a}$ B.S., and Marcela Bailez $z^{b}$ M.D.
}

\begin{abstract}
Purpose. The objective was to describe health related quality of life (HRQoL) after surgery for Hirschsprung's disease (HD) and anorectal malformations (ARMs) in children according to their own perception and that of their parents, by different age groups, the presence of functional disorders, and diet indication.

Methods. This is a cross-sectional descriptive study. Participants were patients aged 2-18 years old and their parents. The study was conducted from June 2008 to May 2009. Theinstrument used was the PedsQL ${ }^{\mathrm{TM}}$ 4.0. Data were analyzed with SPSS version 11.5.
\end{abstract}

Results. A total of 93 families gave their consent to participate. Higher scores indicated a better HRQoL. Parents showed a trend towards obtaining higher scores in the three scales analyzed. There were no differences observed in the various age groups. The lowest scores were obtained in incontinent, pseudo-incontinent and constipated patients with statistically significant differences according to the parents' reports when compared to the patients who had no functional disorders. When analyzing dietary therapy, patients who were on a controlled protein and low sodium diet together with a suitable intestinal function obtained lower scores.

Conclusion. The presence of postoperative functional disorders had a negative impact on the scores. Incontinent, pseudo-incontinent and constipated patients had the lowest scores, as well as those who had a stricter diet.

Key words: Health related quality of life, Hirschsprung's disease, anorectal malformations, diets, bowel function.

http:/ /dx.doi.org/10.5546/aap.2013.37

\section{INTRODUCTION}

Hirschsprung's disease or congenital aganglionic megacolon (CAM) is a congenital colon innervation defect. ${ }^{1-3}$

Anorectal malformations (ARMs) include any congenital caudal anomalies accompanying imperforate anus.

Treatment for both conditions has always been surgery.., 5

Given the multiple advances in surgery, post-operative results are satisfactory, but some patients continue to have intestinal dysfunction, such as stenosis, incontinence, soiling, abdominal bloating, constipation, diarrhea and enterocolitis, and some-times, a combination of these. ${ }^{6,7}$

More attention is now being paid to the quality of life of children living with chronic conditions.

"Health-related quality of life (HRQoL) refers to the impact of health and disease or its treatment on the quality of life of an individual". ${ }^{9}$ It constitutes a unique personal concept, a subjective perception of how an individual feels about his/her health status and the non-medical aspects of his/her life. ${ }^{10}$

The PedsQL is an instrument developed by James Varni in USA in the 1990s. It is a generic module designed to measure HRQoL in healthy and acutely or chronically ill children and adolescents. It is a 23-item measurement model, for health-related quality of life in children aged 2 to 18 years and has a self-report form and a parent's proxy-report form. It includes physical, emotional, social and school functionning. Given its psychometric properties, it is recommended for clinical use and to compare groups. The author of the questionnaire has granted Hospital Garrahan a license to be used for scientific purposes. ${ }^{9,12,13}$ The Quality of Life and Health Committee of our hospital has localized and validated a version of this instrument in Spanish.

Most long-term studies deal with functional results, and some of them have shown how disease-specific problems impact mental health and psychosocial functioning, but the quality of life of these patients has been poorly studied. ${ }^{6,8}$ 
A study in children after surgery of ARMs reported problems related with peers, school absenteeism, restrictive diets and behavioral problems. Quality of life was lower in patients when compared with controls, and lower in incontinent children. ${ }^{14}$ Similar results were found long term after the Swenson procedure for Hirschsprung's disease. $^{6}$

A publication about children with chronic constipation studied with the PedsQL ${ }^{\mathrm{tm}}$ showed lower HRQoL scores for these patients than for children with inflammatory bowel disease, gastroesophageal reflux or healthy children. ${ }^{15}$

The objective of this article is to describe HRQoL in children and adolescents following a definitive surgery for CAM and ARMs, according to their own perception and that of their parents' using a generic instrument.

Also, to assess HRQoL by age group and observe any trends in results according to the presence of complications and whether they have an indication for diet and what type.

\section{POPULATION AND METHODS}

This was a descriptive, cross-sectional study.

The study population included children and adolescents followed-up by the Colorectal Unit of Hospital de Pediatría "Prof. Dr. Juan P. Garrahan" that attended a control visit between July 2008 and May 2009. Patients who met the inclusion criteria, and their parents, were invited to participate in the study. The questionnaire was administered at the doctor's office by a member of the health team, a B.S. in Nutrition, prior to the appointment at the Colorectal Unit.

The questionnaire was provided according to the PedsQL ${ }^{\mathrm{tm}}$ Administration Guidelines. ${ }^{16}$

Children aged 2-18 years old were included in the study. All of them had undergone a definitive surgery, and three or more months had elapsed since the surgery. Patients were excluded if they had associated pathologies, except for urological and spine conditions, serious cognitive impairment and less than three months after surgery.

In order to participate, the father, mother or caregiver had to provide his/her signed informed consent and children older than 10 years old had to give their consent.

The sample was selected by convenience, in a sequential, non-randomized manner.

The following endpoints were collected directly from the patients' medical records on the same day the questionnaire was applied: age (as per the four PedsQL age groups), gender, diagnosis
(CAM-ARM), presence of functional symptoms, diet and time since surgery.

For the analysis of this sample, post-operative functional sequelae were divided into four categories: A) no post-operative functional disorders; B) diarrhea: more than three liquid bowel movements per day; C) incontinence and soiling; and D) constipation: bowel movement with symptoms indicative of pain or discomfort and abdominal bloating, generally associated with passing very hard stools.

Group " $\mathrm{C}$ " includes patients with both true incontinence and soiling: soiling in a patient who had developed voluntary defecation during the post-operative period is a serious constipation symptom; leakage of liquid stool material is caused by rectal impaction. Incontinence occurs in patients with involuntary bowel movements and soiling. ${ }^{5}$ For analysis purposes, these conditions are considered as only one group, defined as involuntary passage of stool.

Diets were classified as follows: A) no restriction diet; B) low-fermentation diet: lactose-, sucrose- and cellulosic residue-low diet; C) highfiber diet: increased intake of cellulosic residue; D) low-fermentation diet with sodium and proteins according to recommendations; and E) highfiber diet with sodium and proteins according to recommendations.

For the validation of the instrument, a sample of healthy children and adolescents was used as reference population in this study; it was composed of 105 children and adolescents attending one of the three children's hospitals in the city of Buenos Aires and a primary school in Greater Buenos Aires. Socio-demographic characteristics of these children were similar to those of children with chronic conditions receiving care at the hospital. ${ }^{11}$

The project was approved by the hospital's Research and Teaching Board and Ethics Committee.

\section{Analysis Schedule}

According to PedsQL ${ }^{\mathrm{tm}}$ instructions, a questionnaire with $50 \%$ or more completed and validated questions in each domain was considered valid for the total score.

In order to make interpretation of data easier, the scale of results may be converted from a $0-4$ scale into a $0-100$ scale. ${ }^{17}$

Results are reported as a total score and, at the same time, by psychosocial and physical domains (emotional, social and school) separately.

All results are reported as mean values and 
standard deviations, or as median values and ranges, depending on the endpoint distribution. In terms of group differences, a t test for independent samples with normal distribution was used for their assessment, and when the distribution was not parametric, a Wilcoxon test was used.

When there were more than two groups, differences were assessed using a statistical ANOVA test. A $p$ value of $\leq 0.05$ was considered statistically significant.

All data were processed using the statistical package SPSS11.5.

\section{RESULTS}

\section{Descriptive results}

A total of 93 children and their parents participated. The sample accounts for $21 \%$ of patients that had an appointment. The most frequent causes for the lack of participation of patients were: younger than 2 years old, recently operated, presence of associated disorders or an ileostomy or colostomy.

The questionnaire was easy to apply; most patients and their parents did not require help, only $6 \%$ of the patients required assistance.

Table 1 describes population characteristics according to the study endpoints.

It shows how functional disorders improve with age. In the group of adolescents, functional disorders reach $47.1 \%$ without intestinal dysfunction (Table 1). In the 2-4 year old group, it was not possible to assess continence, except in children older than 3.5 years old.

At the time of the survey, $56 \%$ of patients had some kind of diet indication. It should be noted that the remaining $44 \%$ included some patients that did require a diet.

TABLE 1. Population characteristics according to the study endpoints

\begin{tabular}{|c|c|c|c|c|c|}
\hline Age group & 2-4 years old & 5-7 years old & 8-12 years old & 13-18 years old & Total \\
\hline Total & $24(25.8 \%)$ & $26(28 \%)$ & $26(28 \%)$ & $17(18.3)$ & $93(100 \%)$ \\
\hline \multicolumn{6}{|l|}{ Gender } \\
\hline Male & $14(58.3 \%)$ & $15(57.7 \%)$ & $16(61.5 \%)$ & $7(41.2 \%)$ & $52(55.9 \%)$ \\
\hline Female & $10(41.7 \%)$ & $11(42.3 \%)$ & $10(38.5 \%)$ & $10(58.8 \%)$ & $41(44.1 \%)$ \\
\hline \multicolumn{6}{|l|}{ Diagnosis } \\
\hline Hirschsprung's disease & $12(50 \%)$ & $6(23.1 \%)$ & $7(26.9 \%)$ & $5(29.4 \%)$ & $30(32.3 \%)$ \\
\hline Anorectal malformations & $12(50 \%)$ & $20(76.9 \%)$ & $19(73.1 \%)$ & $12(70.6 \%)$ & $63(67.7 \%)$ \\
\hline \multicolumn{6}{|c|}{ Post-operative functional disorders } \\
\hline No disorders & $6(25.0 \%)$ & $3(11.5 \%)$ & $6(23.1 \%)$ & $8(47.1 \%)$ & $23(24.7 \%)$ \\
\hline Diarrhea & $9(37.5 \%)$ & $4(15.4 \%)$ & $1(3.8 \%)$ & $1(5.9 \%)$ & $15(16.1 \%)$ \\
\hline Incontinence or soiling & $4(16.7 \%)$ & $15(57.7 \%)$ & $11(42.3 \%)$ & $5(29.4 \%)$ & $35(37.6 \%)$ \\
\hline Constipation & $5(20.8 \%)$ & $4(15.4 \%)$ & $8(30.8 \%)$ & $3(17.6 \%)$ & $20(21.5 \%)$ \\
\hline \multicolumn{6}{|l|}{ Diets } \\
\hline $\mathrm{A}$ & $11(45.8 \%)$ & $7(26.9 \%)$ & $12(46.2 \%)$ & $11(64.7 \%)$ & $41(44.1 \%)$ \\
\hline B & $9(37.5 \%)$ & $7(26.9 \%)$ & $2(7.71 \%)$ & $1(5.9 \%)$ & $19(20.4 \%)$ \\
\hline $\mathrm{C}$ & $2(8.3 \%)$ & $6(23.1 \%)$ & $7(26.9 \%)$ & $2(11.8 \%)$ & $17(18.3 \%)$ \\
\hline $\mathrm{D}$ & $1(4.2 \%)$ & $2(7.7 \%)$ & & $1(5.9 \%)$ & $4(4.3 \%)$ \\
\hline $\mathrm{E}$ & $1(4.2 \%)$ & $4(15.4 \%)$ & $5(19.2 \%)$ & $2(11.8 \%)$ & $12(12.9 \%)$ \\
\hline \multicolumn{6}{|l|}{ Time since surgery } \\
\hline 3-12 months & $7(29.2 \%)$ & $1(3.8 \%)$ & $1(3.8 \%)$ & $2(11.8 \%)$ & $11(11.8 \%)$ \\
\hline 13-36 months & $13(54.2 \%)$ & $4(15.4 \%)$ & $1(3.8 \%)$ & $1(5.9 \%)$ & $19(20.4 \%)$ \\
\hline More than 36 months & $4(16.7 \%)$ & $21(80.8 \%)$ & $24(92.3 \%)$ & $14(82.4 \%)$ & $63(67.74 \%)$ \\
\hline
\end{tabular}

Diet classification:

A: No restriction diet.

B: Low-fermentation diet: lactose-, sucrose- and cellulosic residue-low diet.

C: High-fiber diet: increased cellulosic residue diet.

D: Low-fermentation diet with protein restriction and low sodium: lactose-, sucrose- and cellulosic residue-low diet, proteins and sodium according to recommendation.

E:High-fiber diet with protein and sodium restriction: high-cellulosic residuediet, proteins and sodium according to recommendation. 
In terms of the time since surgery, more than $60 \%$ of patients included in the sample had undergone surgery more than 36 months before.

In relation to urinary incontinence, ARM patients were: one incontinent patient, one patient with occasional and nocturnal incontinence, and seven patients submitted to Mitrofanoff's technique to achieve continence.

\section{Statistical Results}

When analyzed as a whole, parents' scores seemed higher than those of children. Statistical differences between parents and children were observed only in the physical domain using a Wilcoxon test, with $p=0.017$ (Table 2).

In terms of the different age groups, statistical differences were found only in the psychosocial domain of parents' reports, with a $p=0.043$ using an ANOVA test. No linear correlation was found either between scores and age analyzed as a continuous endpoint (Table 3).
When the influence of the presence or absence of post-operative functional disorders on scores was analyzed (as two groups), we observed that both children and their parents had the highest scores if they had no post-operative functional disorder, and scores were statistically significant in the three domains analyzed by parents (Wilcoxon test) (Table 4-A).

HRQoL scores were lower in children and parents with soiling, incontinence or constipation, with statistically significant differences according to parents (Table 4-B).

The analysis of differences among scores of patients with post-operative functional disorders indicated that the lowest scores corresponded to children with incontinence, soiling or constipation, and the highest scores were found in those with diarrhea, but no statistically significant difference was obtained.

In terms of how having or not having a diet indication influenced scores (as two groups), both

TABLE 2. Children's and parents' scores (median and range)

\begin{tabular}{|c|c|}
\hline & Scores \\
\hline Children's and adolescents' report & Median (range) \\
\hline \multicolumn{2}{|l|}{$\mathrm{N}: 43$} \\
\hline Total score & $78.26(59.78-95.65)$ \\
\hline Physical domain score & $87.5(46.87-100)$ * \\
\hline Psychosocial domain score & $78.33(45-98.33)$ \\
\hline Emotional domain score & $75(40-100)$ \\
\hline Social domain score & $85(45-100)$ \\
\hline School domain score & $75(40-100)$ \\
\hline \multicolumn{2}{|l|}{ Parents' report } \\
\hline \multicolumn{2}{|l|}{ N: 93} \\
\hline Total score & $86.11(31.81-100)$ \\
\hline Physical domain score & $93.75(37.5-100)$ * \\
\hline Psychosocial domain score & $82.5(28.57-100)$ \\
\hline Emotional domain score & $75(10-100)$ \\
\hline Social domain score & $90(45-100)$ \\
\hline School domain score & $75(5-100)$ \\
\hline
\end{tabular}

*: Wilcoxon test with a $\mathrm{p}$ value $=0.017$.

TABLE 3. Score by age group (median and range)

\begin{tabular}{lcccc}
\hline Age groups & 2-4 years old & 5-7 years old & 8-12 years old & 13-18 years old \\
\hline $\mathbf{n}$ & 24 & 26 & 26 & 17 \\
$\begin{array}{l}\text { Children's and adolescents' report } \\
\quad \text { Total score }\end{array}$ & NA & NA & $78.26(60.86-95.65)$ & $78.26(59.78-94.56)$ \\
$\quad$ Physical domain score & NA & NA & $87.5(59.37-100)$ & $81.25(46.87-100)$ \\
$\quad$ Psychosocial domain score & NA & NA & $78.33(45-93.33)$ & $76.66(60-98.33)$ \\
Parents' report & & & & \\
$\quad$ Total score & $88.88(68.05-100)$ & $84.15(31.81-98.8)$ & $84.45(56.52-95.65)$ & $82.6(42.39-100)$ \\
$\quad$ Physical domain score & $93.75(65.62-100)$ & $90.62(37.5-100)$ & $90.62(59.37-100)$ & $93.75(37.5-100)$ \\
$\quad$ Psychosocial domain score & $87.5(70-100)^{*}$ & $80.62(28.57-98.33)^{*}$ & $79.16(45-95)^{*}$ & $83.33(45-100)^{*}$ \\
\hline
\end{tabular}

NA: not applicable.

* Psychosocial domain according to parents, with a $\mathrm{p}$ value $=0.043$ with an ANOVA test. 
children and parents had higher scores when there was no diet restriction, and scores were statistically significant in the three scales analyzed according to parents' reports (Table 5- $A$ ).

Table 5- $B$ describes scores by diet indication and type of diet.

A trend towards having lower scores was observed with a more restrictive diet. Statistically significant differences where found when comparing patients with no diet restriction to those with a high fiber diet with protein control and low sodium consumption in the three domains analyzed by parents (total score $p=0.004$, psychosocial domain score $p=0.002$, physical domain score $p=0.029$ using an ANOVA test).

In terms of time since the definitive surgery, scores were comparatively lower in those with less than one year after the surgery in children's reports, but not in those of parents. The assessment of the presence of statistically significant differences in the physical domain indicated that these were only found in children's report: more than 37 months vs. 12 months or less, $p=0.025$ (ANOVA).
Table 6 describes overall scores of patients with surgery for CAM and ARMs, and the scores of healthy children. When analyzed together, the scores of patients undergoing a CAM or ARM surgery seemed higher than those of healthy children. Statistical differences were observed for the total and physical domains in children using a Wilcoxon test, with $p=0.035$ and 0.017 , respectively.

\section{DISCUSSION}

It is possible to assess HRQoL in children with CAM and ARMs with a locally validated generic instrument.

Patients and their parents appeared to be excited about taking part and with the fact that the health team was interested in evaluating their quality of life. Only one adolescent patient that met the inclusion criteria did not give his consent to participate by saying: "I do not feel like doing it today."

In addition, patients and their parents considered that the questions included in the questionnaire were relevant.

TABLE 4-A. Score by presence or absence of post-operative functional disorders - Wilcoxon test

\begin{tabular}{lccc}
\hline & No disorders & With disorder & Mann-Whitney U test \\
\hline $\mathbf{n}$ & 23 & 70 & $\boldsymbol{P}$ \\
Children & Median (range) & Median (range) & 0.169 \\
$\quad$ Total score & $85.32(60.86-94.54)$ & $78.26(59.78-95.65)$ & 0.264 \\
$\quad$ Physical domain score & $87.5(62.5-100)$ & $84.37(46.87-100)$ & 0.326 \\
$\quad$ Psychosocial domain score & $81.66(60-98.33)$ & $76.66(45-93.33)$ & 0 \\
Parents & & & 0 \\
$\quad$ Total score & $92.04(63.04-100)$ & $83.69(31.81-98.61)$ & 0.006 \\
$\quad$ Physical domain score & $93.75(75-100)$ & $90.62(37.5-100)$ & $80(28.57-100)$ \\
$\quad$ Psychosocial domain score & $90(56.66-100)$ & & \\
\hline
\end{tabular}

TABLE 4-B. Comparison of score by presence and type or absence of post-operative functional disorders (median and range)

\begin{tabular}{|c|c|c|c|c|}
\hline & No disorders-A & Diarrhea-B & Incontinence and soiling-C & Constipation-D \\
\hline $\mathbf{n}$ & 23 & 15 & 35 & 20 \\
\hline \multicolumn{5}{|c|}{ Children's and adolescents' report } \\
\hline Total score & $85.32(60.86-94.56)$ & $89.12(82.6-95.65)$ & $78.26(60.86-95.65)$ & 70.65 (59.78-91.3) \\
\hline Physical domain score & $87.5(62.5-100)$ & $90.62(81.25-100)$ & $89.06(62.5-100)$ & $78.12(46.87-96.87)$ \\
\hline Psychosocial domain & $81.66(60-98.33)$ & $88.33(83.33-93.33)$ & $77.49(45-93.33)$ & $73.33(61.66-91.66)$ \\
\hline \multicolumn{5}{|l|}{ Parents' report } \\
\hline Total score & $92.04(63.04-100)^{*}$ & $84.78(68.05-98.61)$ & $82.6(47.82-97.82)^{*}$ & $83.69(31.81-97.22)^{*}$ \\
\hline Physical domain score & $93.75(75-100)^{* *}$ & $87.5(64.28-100)$ & $93.75(53.12-100)$ & $87.5(37.5-100)^{* *}$ \\
\hline Psychosocial domain & $90(56.66-100)^{* * *}$ & $82.5(70-100)$ & $78.33(45-98.33)$ & $79.99(28.57-97.5)^{* * *}$ \\
\hline
\end{tabular}

Analysis of variance ANOVA.

*Parents' total score: no functional disorders/incontinence, no functional disorders/constipation; $\mathrm{p}=0.003$.

**Parents' physical domain score: no functional disorders/constipation; $\mathrm{p}=0.028$.

***Parents' psychosocial domain score: no functional disorders $/$ incontinence, no functional disorders $/$ constipation; $\mathrm{p}=0.002$. 
It should be noted that since the questionnaire was administered to patients during a follow-up visit, most study participants had post-operative functional disorders and were receiving some kind of treatment to improve their intestinal function. Treatments included: education on bowel habits, diet, medications, enemas or the use of a device for antegrade enemas with a percutaneous catheter in the cecum, depending on the patient's individual needs.

HRQoL scores of children with CAM and ARMs who underwent a surgery were, altogether, similar or higher to those of healthy children. When comparing our sample with the sample of healthy children used to validate the instrument, no overall impairment of HRQoL was observed. Hartman et al. found similar results in patients with CAM and ARMs. They believe it is possible that, when compared with healthy adolescents, adolescents with CAM and ARMs have developed stronger psychosocial strategies and capabilities, probably because they had to learn to live with chronic functional problems. ${ }^{18}$
In this population, it is important to establish the factors that did have an influence on HRQoL low scores.

The analysis of HRQoL scores showed that parents had a trend towards having higher scores in the three scales analyzed.

No differences by age group were observed.

Post-operative functional disorders improve with age. The analysis of HRQoL scores in relation to post-operative functional disorders indicates that their presence has a negative impact on scores. The lowest scores were obtained in incontinent, pseudo-incontinent and constipated patients, with statistically significant differences according to their parents when compared with patients with no functional disorders. Studies conducted in constipated patients ${ }^{14-19}$ and in patients with surgery for $\mathrm{CAM}^{20}$ obtained similar results with the same instrument. Fecal incontinence and constipation have a strong impact on the psychosocial aspects of children and adolescents. Studies suggest that there is a strong association between incon-

TABLE 5-A. Score by indication of diet - Wilcoxon test

\begin{tabular}{lccc}
\hline & No diet & Diet & Mann-Whitney U test \\
\hline $\mathbf{n}$ & 41 & 52 & $\mathbf{P}$ \\
Children & Median (range) & Median (range) & 0.116 \\
$\quad$ Total score & $81.52(60.86-95.65)$ & $74.45(59.78-95.65)$ & 0.214 \\
Physical domain score & $80(60-98.33)$ & $82.81(46.87-100)$ & 0.188 \\
$\quad$ Psychosocial domain score & $87.5(65.5-100)$ & $74.16(45-93.33)$ & 0.002 \\
Parents & & & 0.002 \\
$\quad$ Total score & $89.13(57.6-100)$ & $83.69(31.81-98.61)$ & 0.023 \\
Physical domain score & $93.75(59.37-100)$ & $90.62(37.5-100)$ & $79.16(28.57-100)$ \\
$\quad$ Psychosocial domain score & $87.5(56.66-100)$ & & \\
\hline
\end{tabular}

TABLE 5-B. Score by type of diet (median and range)

\begin{tabular}{lccccc}
\hline Dietas & $\begin{array}{c}\text { A } \\
\text { Median (range) }\end{array}$ & $\begin{array}{c}\text { B } \\
\text { Median (range) }\end{array}$ & $\begin{array}{c}\text { C } \\
\text { Median (range) }\end{array}$ & $\begin{array}{c}\text { D } \\
\text { Median (range) }\end{array}$ & $\begin{array}{c}\text { E } \\
\text { Median (range) }\end{array}$ \\
\hline Children's and adolescents' report & 41 & & & & \\
$\quad \mathbf{n}$ & & 19 & 17 & 4 & 12 \\
Total score & $81.52(60.86-95.65)$ & $82.6(73.91-95.65)$ & $79.34(59.78-91.3)$ & $71.33(71.73-71.73)$ & $70.65(60.86-95.65)$ \\
Physical domain & $87.5(62.5-100)$ & $87.5(81.25-100)$ & $84.37(46.87-96.87)$ & $68.75(68.75-68.75)$ & $81.25(59.37-100)$ \\
Psychosocial domain & $80(60-98.33)$ & $83.33(66.66-93.33)$ & $78.33(45-91.66)$ & $73.33(73.33-73.33)$ & $66.66(60-93.33)$ \\
Parents' report & & & & & \\
Total score & $89.13(57.6-100)^{*}$ & $84.78(61.36-98.61)$ & $83.69(42.39-95.65)$ & $80.38(68.47-91.66)$ & $71.19(31.81-93.05)^{*}$ \\
Physical domain & $93.75(59.37-100)^{* *}$ & $90(64.28-100)$ & $93.75(37.5-100)$ & $95.31(89.28-100)$ & $82.81(37.5-100)^{* * *}$ \\
Psychosocial domain & $87.5(56.66-100)^{* * *}$ & $81.66(48.21-100)$ & $78.33(45-93.33)$ & $73.33(56.66-85)$ & $64.99(28.57-93.33)^{* * *}$ \\
\hline
\end{tabular}

ANOVA analysis of variance.

*Parents' total score: no diet restriction/high-fiber diet with protein control and low-sodium consumption; $\mathrm{p}=0.004$.

**Parents' physical domain score: no diet restriction/high-fiber diet with protein control and low-sodium consumption; $\mathrm{p}=0.029$.

***Parents' psychosocial domain score: no diet restriction/high-fiber diet with protein control and low-sodium consumption; $\mathrm{p}=0.002$. 
tinence and its negative effects on activities and social life of children aged 5-15 years old..$^{21-23}$

A review indicates that, based on the few available studies, it may be concluded that intestinal function is better in adolescents than in children, while adolescents tend to have worse psychosocial problems in their HRQoL. ${ }^{24}$

It is difficult to assess whether a dietary therapy intervention improves HRQoL in children and adolescents who had surgery for CAM and ARM, since patients with post-operative functional disorders are the ones who had a diet indication. When analyzing patients with no diet restriction in relation to those with dietary therapy, higher scores in children with no diet restriction were found with statistically significant differences in the three scales analyzed according to their parents. The analysis of dietary therapy indication showed that patients who had a "protein-controlled and low-sodium diet" at the time of filling in the questionnaire in addition to adequate intestinal function had the lowest scores. It should be noted that these are patients with more complex ARMs and urological or kidney involvement. Higher scores were obtained in patients with a low-fermentation diet or a high-fiber diet. One of the study limitations is that, being a cross-sectional study, it was not possible to assess dietary therapy intervention in the same patient. Conducting a longitudinal study with the questionnaire administered before and after the intervention in the same patient would allow researchers to evaluate its impact on HRQoL.

\section{CONCLUSIONS}

It is important to perform a long term multidisciplinary follow-up of patients throughout their development. While intestinal function improves with time, there is still a group of school-aged children and adolescents who have intestinal function disorders. The health team should identify such problems and improve the selection and development of treatment strategies targeted at improving quality of life.

The use of the "PedsQ1" instrument in the doctor's office would allow professionals to find out which problems are relevant to each patient and help them with making decisions. Taking the perspective of children and their parents into consideration in relation to the patient's health status and the psychosocial aspects at the time of their visit to the doctor may improve and help with treatment compliance.

\section{Acknowledgments}

This study was conducted thanks to the research fellowship granted by the "Fundación Hospital de Pediatría Prof. Dr. Juan P. Garrahan".

Food Area: to the head of the area, Sandra Blasi, B.S., and her assistants, Alice Du Mortier, B.S., Mercedes Pico, B.S., and Silvana Lavorgna, B.S., for their continuous help and assistance.

To the members of the "Colorectal Unit", composed of professionals from the surgery, clinical, nursing and gastroenterology services, especially to Julieta Solanas, M.D., Mariana Nastri, M.D. and Víctor Di Benedetto, M.D.

It was very important to have the guidance and helpfulness of the Teaching and Research Associate Board. The collaboration of Mariana Roizen, M.D., was also important in the validation of the instrument we used and provided her guidance on the instrument and its statistics.

\section{BIBLIOGRAPHY}

1. Swenson O. Hirschsprung's disease: A review. Pediatrics 2002;109:914-8.

2. Tojo R. Tratado de Nutrición Pediátrica. Barcelona: Ediciones Doyma; 2001.Págs.807-23.

3. Sociedad Española de Gastroenterología, Hepatología y Nutrición Pediátrica. Tratamiento en Gastroenterología,

TABLE 6. Healthy and CAM-ARM children scores (median and range)

\begin{tabular}{lcc}
\hline Population & Healthy children & CAM and ARM children \\
\hline $\mathbf{n}$ & 105 & 93 \\
Children's and adolescents' report & & $78.26(59.78-95.65)$ \\
$\quad$ Total score & $72.82(36.96-95.65)$ & $87.5(46.87-100)$ \\
$\quad$ Physical domain score & $75(18.75-100)$ & $78.33(45-98.33)$ \\
$\quad$ Psychosocial domain score & $71.66(31.67-95)$ & \\
Informe para padres & & $86.11(31.81-100)$ \\
$\quad$ Total score & $83.69(43.48-100)$ & $93.75(37.5-100)$ \\
$\quad$ Physical domain score & $87.5(50-100)$ & $82.5(28.57-100)$ \\
$\quad$ Psychosocial domain score & $82.69(40-100)$ & \\
\hline
\end{tabular}


Hepatología y Nutrición Pediátrica. Madrid: Ergon. C/ Arboleda; 2004. Págs.122-6.

4. Di Lorenzo C, Benninga MA. Fisiopatología de la incontinencia fecal pediátrica. Gastroenterology 2004;126:33-40.

5. Bailez M, Prieto F. Malformaciones ano-rectales. Enciclopedia Médica Americana. Cir Dig 2007;364:1-19.

6. Bai Y, Chen H, Hao J, Huang Y, et al. Long-term outcome and quality of life after the Swenson procedure for Hirschsprung's disease. J Pediatr Surg 2002;37:639-42.

7. Tsuji H, Spitz L, Kiely EM, Drake DP, et al. Management and long-term follow-up of infants with total colonic aganglionosis. J Pediatr Surg 1999;34:158-62.

8. Dasgupta R, Langer J. Evaluation and management of persistent problems after surgery for Hirschsprung's disease in a child. J Pediatr Gastroenterol Nutr 2008;46:13-19.

9. Comité de Calidad de Vida Relacionada a la Salud. Hospital de Pediatría "Prof. Dr. Juan P. Garrahan". Calidad de vida relacionada a la salud. Med Infant 2004;11:301-5.

10. Barnes P, Jenney M. Measuring quality of life. Curr Paediatr 2002;12:476-80.

11. Roizen M, Rodríguez S, Bauer G, Medin G, et al. Initial validation of the Argentinean Spanish version of the Ped$\mathrm{sQL}^{\mathrm{TM}} 4.0$ Generic Core Scale in children and adolescents with chronic disease: Acceptability and comprehensibility in low income settings. Health Qual Life Outcomes 2008;6:59.

12. Roizen M, Figueroa C, Salvia L y miembros del Comité de Calidad de Vida y Salud. Calidad de vida relacionada con la salud en niños con enfermedades crónicas: comparación de la visión de los niños, sus padres y sus médicos. Arch Argent Pediatr 2007;105(4):305-13.

13. PedsQl ${ }^{\mathrm{TM}}$ Website [Accessed on: April 30, 2008]. Available at: http//www.pedsql.org/about_pedsql.html.

14. Bai Y, Yuan Z, Wang W, Zhao Y, et al. Quality of life for children with fecal incontinence after surgically correct- ed anorectal malformation. J Pediatr Gastroenterol Nutr 2007;44:540-54

15. Youssef N, Langseder A, Verga B, Mones R, et al. Chronic childhood constipation is associated with impaired quality of life: a case-controlled study. J Pediatr Gastroenterol Nutr 2005;41:56-60.

16. Varni W. (2008). PedsQl ${ }^{\mathrm{TM}}$ Administration Guidelines. [Accessed on: May 7, 2008]. Available at: http//www. pedsql. org/pedsqladmin.html.

17. Varni W. (2008). PedsQ1 ${ }^{\mathrm{TM}}$ Scoring Algorithm. [Accessed on: May 7, 2008].Available at: http//www.pedsql.org/ score.html.

18. Hartman E, Oort F, Sprangers M, et al. Factors affecting quality of life of children and adolescents with anorectal malformation or Hirschsprung disease. J Pediatr Gastroenterol Nutr 2008;47:463-71.

19. Clarke M, Chow C, Chase J, Gibb S, et al. Quality of life in children with slow transit constipation. J Pediatr Surg 2008;43:320-4.

20. Mills J,Konkin D, Milner R, Penner J, etal. Long-term bowel function and quality of life in children with Hirschsprung disease. J Pediatr Surg 2008;43:899-905.

21. Yanchar N,Soucy P. Long-term outcome after Hirschsprung disease: patients perspectives. J Pediatr Surg 1999;34:115260.

22. Eiser C, Jenney M. Measuring quality of life. Arch Dis Child 2007;92:348-50.

23. Kuyk E, Brugman-Boezeman A, Wissink-Essink M, Severijnen $R$, et al. Defecation problems in children with Hirschsprung disease: a biopsychosocial approach. PediatrSurg Int 2000;16:312-16.

24. Hartman E, Oort F, Aronson D, Sprangers M. Quality of life and disease-specific functioning of patients with anorectal malformations or Hirschsprung disease: a review. Arch Dis Child 2011;96:398-406. 